\section{UCDNN}

LIBRARY
University of Connecticut OpenCommons@UConn

Philosophy Articles

Philosophy Department

September 1999

\title{
Ontology and Realism about Modality
}

Crawford Elder

University of Connecticut Department of Philosophy, crawford.elder@uconn.edu

Follow this and additional works at: https://opencommons.uconn.edu/philo_articles

\section{Recommended Citation}

Elder, Crawford, "Ontology and Realism about Modality" (1999). Philosophy Articles. 7. https://opencommons.uconn.edu/philo_articles/7 


\section{ONTOLOGY AND REALISM ABOUT MODALITY}

Crawford L. Elder

To be a realist about modality, need one claim that more exists than just the various objects and properties that populate the world-e.g. worlds other than the actual one, or maximal consistent sets of propositions? Or does the existence of objects and properties by itself involve the obtaining of necessities (and possibilities) in re? The latter position is now unpopular but not unfamiliar. Aristotle held that objects have essences, and hence necessarily have certain properties. Recently it has been argued that the identity of any property is tied to the natural laws in which it figures, which entails that the occurrence of properties involves the obtaining of nomological necessities ([24], pp. 206-33 and 234-60; [23]; cf. [25] and [6]). Somewhat less recently, Wittgenstein ([28], p. 168) worried that the reality of at least some properties_-precise shades of colors being a prime example-involved the obtaining in re of certain impossibilities.

This paper argues that Wittgenstein's worries were right, and not just concerning some properties, but all properties whatever. That there are objects and properties in the world at all, then, amounts to there obtaining modal states of affairs. This argument supplements, rather than replaces, the others. The position on property incompatibility advanced here actually helps defend Aristotelian essentialism against epistemological objections, or so I have argued ([15]). And while this paper's position on property incompatibility diverges from the idea that a property's nomic profile is essential to it—more on this in the next section-it is at least compatible with the thought that the necessity involved in the laws of nature enters into ontology at the ground level. 
In confirmation of Wittgenstein's worries, this paper argues that any property is flanked by contrary properties, with which it is incompatible, and that this incompatibility is a substantive in re impossibility of joint instantiation. The properties exclude one another not because of how we name them or think about them (that is the 'in re' part), and that each excludes the others does not reduce to the fact that each is, intrinsically, the particular property it is (that is the 'substantive' part-more in §III). All properties are such as Wittgenstein worried particular shades of color-indeed, all properties attributed by 'unanalyzable statements of degree'-were. But I shall not offer colors themselves as an illustration of my thesis. We will consider a variety of sample ranges of properties that are, or appear to be, contraries of one another. For some sample ranges encourage the thought that the incompatibility of contraries is not substantive, or not in re, and so explain the resistance to the position of this paper; others show, if I am right, that that position cannot in the end be avoided. But concerning colors themselves I take recent philosophical work on color vision—notably Austen Clark's ([9], Ch. 6)— to have shown that there are no such properties. There are only color predicates, e.g. 'is red'. The extension of any such predicate is a ragtag rabble of surface reflectance profiles and wavelengths of colored light, not characterized by any genuine objective sameness. In contrast the properties in the following ranges have a fair claim to being genuine, and to being incompatible with one another: weighing $5 \mathrm{lbs}$. versus weighing $2 \mathrm{lbs}$. versus weighing $6 \mathrm{lbs}$; ; being $3 \mathrm{ft}$. in length versus $2 \mathrm{ft}$. or $4 \mathrm{ft}$; having a normal body temperature of $98.6^{\circ} \mathrm{F}$ versus $99.8^{\circ} \mathrm{F}$ versus $94^{\circ} \mathrm{F}$. Or consider properties like these, whose reality I will defend in $\S \mathrm{IV}$, which if real are undoubtedly incompatible with contraries: having a valence of +3 ; developing a maximum horsepower of 409; sending a signal of $n$ volts, as said by a neuroscientist of a particular sensory channel at a time; or having a high degree of stability, as said of a particular combination of alleles in population genetics.

This paper will focus on the mutual incompatibility of the properties in any such range, but more than that is involved in the fact that the properties are contraries of one another. To put it roughly at first, 
the contraries of a given property must be its own proper rivals, relevant alternatives to it, other properties which in a way resemble it. This is indeed crucial to the idea that a property's being incompatible with certain other properties is central to its being itself, to its very identity. For there are incompatibilities aplenty which, intuitively, do not reflect the nature or identity of the properties they join. Having atomic number 79 for example is (on plausible assumptions) incompatible with having an IQ of 128; nothing which has either property can have the other. But this incompatibility seems to be a function of the cognitive limitations of atoms, or of the design restrictions on how small a thinking organ can be engineered. It seems not to reflect what having atomic number 79 amounts to or by nature is like. But that having atomic number 79 by nature rules out a thing's having atomic number 80 or 78 is, in contrast, a plausible surmise.

Just what a property's being a 'proper rival' of a given one really amounts to is, in part, something under dispute between the position of this paper and its opponents. For there are analyses of proper rivalry (see §III) which entail that the incompatibility of contraries is not a substantive impossibility at all, but just a function of what each of the contraries intrinsically amounts to. There is however a sharpening of the concept which is neutral for this debate, and it is a useful one. The properties in any one range of contraries contrast with all the other properties in the range, and contrast with them to different—but commensurable—degrees. Thus anything having an IQ of 128 thereby differs from anything having an IQ of 120, and differs yet more sharply from anything having an IQ of 100. Something which has an IQ of 128 also differs from anything having an atomic number of 16, but this difference is not sharper still, or milder, but a matter of utter lack of connection; and something with IQ 128 differs again from anything having atomic number 25, but differs not less or more sharply from

${ }^{1}$ This account of what contrary properties have in common is taken, with simplifications, from [7], pp. 53-62. It is 'neutral' if taken as a litmus, a formulation of necessary and sufficient conditions, for proper rivalry between a given plurality of properties. See the parenthetical remark at the end of the present paragraph of the text. 
how it differs from the bearers of atomic number 16. One thing which true contraries have in common then, and incompatibles that are not contraries lack, is that each differs differently but commensurably from all the others. (Is differing differently from all those other properties a function of the intrinsic nature of each of the contrary properties—as is argued in [3]—or a relation which ontologically stands on its own feet? In §III I argue that the former answer cannot uniformly be defended. From this it will follow that a property's excluding those others is truly a substantive incompatibility.)

\section{II}

But surely, an opponent of my position might say, it is merely trivial or analytic that there cannot be something which, say, weighs 5 lbs. and also has some other weight. Surely it is just a condition on the practice of ascribing weights at all that anything rightly said to weigh $5 \mathrm{lbs}$. cannot rightly be said to weigh $3 \mathrm{lbs}$. or 10, and so likewise for talk about lengths, normal body temperatures, and the rest. In that case 'necessarily, something which weighs 5 lbs. does not weigh 3 lbs.' voices a merely de dicto necessity. Perhaps indeed, my opponent might add, it is no accident that Wittgenstein's worries about mutual exclusion of properties focused on examples involving colors: perhaps in their case alone does the incompatibility seem not be merely analytic but substantive.

But necessities acknowledged in our very concepts - or in the meanings of the terms we use in reporting the necessities - may at the same time obtain in re. One stock example of a metaphysical necessity in re is that gold has atomic number 79. Possession of that atomic number seems built into the objective nature of that element, that property-bearer; it seems no mere projection of the way we think of gold or of the way we grasp gold with language. But the necessity could for all that be 'analytic'. One can with a little license imagine a hyper-intelligent race for whom the term 'ggold' abbreviates a complex definite description, one conjunct of which attributes atomic number 79, where 'ggold' passes all the relevant tests for referring to gold. Then that gold has atomic number 79 is a genuine necessity, obtaining in re, which counts for creatures with well-attuned concepts as analytic ([16], pp. 432-33). 
Fiction may not even be needed to make the point. Now that a large proportion of English speakers know at least some rudiments of chemical theory, 'Water is H20' is close to functioning as an analytic claim. In the parlance of physicists, 'gravity obeys an inverse-square law' shows all the marks (ill-defined though they are) of an analytic claim and yet reports a substantive nomological necessity.

How indeed could a claim of the form 'necessarily, anything which has property $F$ does not have property $G$ ' fail to say something about how the world is? To be sure, if such a claim incorporates a designator for one of the properties which turns the claim as a whole into a substitution instance of a logical truth—e.g., 'anything which has property $F$ does not have the property of lacking $F$ ' - then indeed the claim is empty and uninformative. But if weighing $5 \mathrm{lbs}$. and weighing $3 \mathrm{lbs}$. are real properties, and distinct from one another (more on their distinctness in the next section), they can be picked out by independent designators - two expressions, each of which maps one of the two properties regardless of whether the other expression maps the other property. The expressions 'weighs 5 lbs.' and 'weighs 3 lbs.' seem precisely to be such designators. 'There can be nothing which weighs 5 lbs. and also weighs 3 lbs.' does not enunciate a merely logical truth.

It is perfectly possible that something which weighs $160 \mathrm{lbs}$. have an IQ of 120 . But it is not possible that something which weighs $160 \mathrm{lbs}$. should weigh $180 \mathrm{lbs}$. Why is this impossible, while the former situation is possible? The explanation must lie in the natures of the properties themselves, weighing $160 \mathrm{lbs}$. and weighing $180 \mathrm{lbs}$. The impossibility must be an impossibility in re.

III

Bishop Butler famously remarked, 'every thing is what it is, and not another thing'. How right he was! It seems hard to deny that even a modally strengthened version of Butler's dictum is true: 'necessarily, every thing is what it is'—read as, is itself, is self-identical—'and it is impossible that any thing be another thing'. But the necessity of self-identity is not enough to establish the position of this paper. That every property necessarily is itself, and cannot be another property, does not amount to a 
substantive necessity in re. For one thing, self-identity may not be a property (or relation) which obtains out there in the world at all ([20], p. 197). In that case, even if it is true that every property must have it (or bear it to itself), no necessity in re has been established. But secondly, even if self-identity is a real property or relation, that every property must have it, and cannot bear it to another property, are not substantive modal truths.

David Armstrong has argued that the incompatibility of any two properties in a contrary range is really just a special case of the second of these modal truths. It really just amounts to the fact that $a$ 's having $F$-where $F$ is a certain sort of complex property - is what it is, and cannot be another thing. For that is how Armstrong proposes to analyze the properties in any contrary range: all of them (or all but one) are complex properties or 'structural universals' ([4], pp. 120-29; [3], pp. 311-15). What it is to have $F$, say, is to comprise a proper part which has property $P_{1}$, another proper part which has $P_{2}$, and a third which has $P_{3}$. The other properties in $F$ 's range are related to $F$ by 'partial identity'. Thus to have $G$, say, is to comprise just two proper parts, one having $P_{1}$ and one having $P_{2}$. The incompatibility of contraries then reduces to what we could call 'the Butler impossibility'. Suppose first-to see the point most easily - that one of $F$ 's contraries is a simple property $H$. Having $H$ then amounts in effect to comprising a part which has $P_{1}$, and no other part. (That is, $H$-ness just is $P_{l}$-ness.) But then of course nothing which

${ }^{2}$ There is actually a line of thought that suggests that these are substantive modal truths. One begins by supposing that every property has its own haecceity (motivated, perhaps, by the apparent intelligibility of the idea that two properties should trade nomic profiles with one another-see [22]). One then surmises that the various haecceities may be naturally incompatible with one another, just as the property of weighing $4 \mathrm{lbs}$. is naturally incompatible with the property of weighing $6 \mathrm{lbs}$. One concludes that it is a substantive natural incompatibility that keeps one property from being another property. But I shall not pursue this line, since on my view haecceities are not properties at all-more precisely, there are no such properties as haecceities. For haecceities do not have contraries. They do not differ, to differing but commensurable degrees, from other haecceities. 
has $F$ can have $H$; having $F$ is comprising a part having $P_{1}$ together with other parts. impossibility is not a substantive one. And the same non-substantive impossibility prevents anything which has $G$ from having $F$. It does not matter if all the properties in a contrary range are complex, so long as they overlap in the right way.

Such an analysis works admirably for the contrariety of such properties as weighing 5 lbs. and weighing 3 lbs., or being $4 \mathrm{ft}$. long versus being $3 \mathrm{ft}$. long, or lasting 10 minutes versus lasting 2 . It even works, as Armstrong has recently shown, for contraries such as being triangular versus being quadrilateral versus being pentagonal, or such as an angle's being right versus acute versus obtuse ([2], pp. 55-56).

But what if there are properties which objects have only as wholes-without their parts having weakened or partial versions of them? Supposing that there are such properties is just supposing that some aggregations (or heaps) of parts may amount to wholes in nature, and not just relative to our classificatory practices. Examples might include-details in a moment-having a valence of +3 , developing a maximum horsepower of 409 , having a normal body temperature of $98.6^{\circ} \mathrm{F}$, or sending a signal of $n$ volts, as said at a time of a particular sensory channel. Such properties might in a sense be styled 'emergent', since they do not materialize until all the parts of their bearers are assembled. But they are not 'emergent' in the mysterious sense in which the vitalists held life (livingness) to be emergent when suitable tissues are suitably arranged. No one denies that science can explain the occurrence of these properties, and explain it by looking at the parts of the property-bearers, and how those parts are arranged. For now I shall assume that there are some such properties; in §IV I will defend the supposition.

${ }^{3}$ So a different way of expressive the non-substantive impossibility here would be to say that a bearer of $F$ cannot be a bearer of $H$-since it is impossible that any thing should be another thing. This is closer to Armstrong's formulation: for $F$ and $H$ 'to qualify the very same particular, that particular would have to be identical with its proper part, which would make it non-identical with itself'([2], p. 54). 
Say then that some internal combustion engine develops a maximum horsepower of 409 . Then it may well comprise a proper part which would develop a lesser horsepower; perhaps if four of the eight cylinders were lopped off, the resulting engine would produced $150 \mathrm{hp}$. But the problem for Armstrong's position is that all the incompatibilities between properties in a given contrary range are to be explained by appeal to partial identities. Consider then the incompatibility between developing $409 \mathrm{hp}$ at the maximum and developing just 407. Armstrong's explanation must be that what it is for something to have a maximum hp of 409 is for it to comprise a part which develops a maximum of 407 together with another part which adds yet more—-specifically, 2 hp more. But it seems impossible to discern, within a $409 \mathrm{hp}$ engine, a proper part which develops 407. If some proper part truly were in its own right responsible for $407 \mathrm{hp}$, it would have to be able to develop $407 \mathrm{hp}$ all by itself-that is, if separated off from the original engine. But the smallest removal of a part from the 409 engine which affects its power at all reduces it to far less than 407. It is even harder to discern, within the 409 engine, the extra bit responsible for the extra $2 \mathrm{hp}$, and for exactly the same reason.

The valence of an element, as originally conceived, concerns the disposition of its atoms to bond with other atoms. Strictly speaking the different valences are incompatible with one another only over the lighter elements, viz. up through scandium on the periodic table; heavier elements (e.g. iron) can have more than one valence (e.g. can bond in either the valence +2 or the valence +3 way). Still, even the heavier elements have only one maximally stable way of bonding, one maximally stable valence. So maximally stable valences are true contraries. If some atom has a (maximally stable) valence of +3 , does it comprise a proper part which has a (maximally stable) valence of +2 , and also a proper part which has a valence of +1 ? Not as a rule. A proper part which had in its own right a valence of +2 or +1 would have to take that valence with it when existing on its own. Yet some ways of breaking pieces off an atom can yield atoms having higher valence. Abstractly, indeed, this can happen in innumerable ways: just move up one row in the periodic table from the original atom, then move right for a proper part having higher positive valence, left for a proper part having higher negative valence. But separation of a higher-valence proper part does also happen in nature. When, for example, sodium-20 undergoes beta-delayed alpha 
emission, what remains is oxygen-16, which has higher positive valence. When beta plus decay happens to carbon-11, what remains is boron-11, and boron has a higher negative valence. 1

Now the development of atomic theory did in time yield a non-dispositional (or less obviously dispositional) understanding of valence, viz. as number of gaps in the outermost electron shell. But the problem here, for the 'partial identity' view, is that electron shells do not in any clear sense have parts. If an outermost electron shell has three gaps, it does not follow that some part of that shell has two gaps.

Third, consider Canis familiaris, whose normal body temperature ranges from $102.2^{\circ}$ to $103^{\circ} \mathrm{F}$. Does a dog comprise a proper part (or parts) the normal temperature of which is, say, $98.6^{\circ} \mathrm{F}$, and another proper part the normal temperature of which is $92^{\circ} \mathrm{F}$, etc.? The main point here is parallel to the point about engines. It is very unlikely that parts can be found within the dog which instantiate all of the available normal body temperatures (or temperature ranges) with which the dog's normal temperature is incompatible. The secondary point is that there is a deep reason why this is unlikely. At root the reason is that 'normal' body temperature does not mean 'statistically average'. One could not reduce the normal body temperature of humans by subjecting three billion of us to severe hypothermia. 'Normal' rather means biologically normal—what the internal temperature has been, on the occasions of historical success which led to selection of the internal devices that produce and that function in that temperature. Now there is controversy whether evolution should be seen as selecting genes and genomes that code for such devices, or the phenotypes that include them, or the populations that display them. But however evolution laid hold of ancestor dogs, in fashioning the dogs of today, it ended up grabbing whole dogs. For evolution operates only by surveying and reproductively rewarding behavior by whole individual organisms. So there is no biologically normal temperature for proper parts of dogs other than the temperature biologically normal for whole dogs. True enough, different temperatures can be biologically normal for different parts: in male dogs, for example, the testes must be cooler than the core. But the requirement of placement in whole living dogs limits severely which differences in normal temperature

\footnotetext{
${ }^{4}$ Encyclopaedia Britannica, 15 ${ }^{\text {th }}$ edn. (1974), Vol. 15, pp. 436 and 438.
} 
can obtain. Parts cannot be discerned ad libitum which have, or even could have, normal temperatures different from $102.2^{\circ}-103^{\circ} \mathrm{F}$.

The final example. A sensory signal in a particular neural channel represents the environment as being a certain way (pace [1]; see my [12]). For that reason, not just any transmission of electrical energy along a neural channel qualifies as a sensory signal: a transmission sparked by a lightning strike does not, and neither does 'noise' generated by random firings of the neurons themselves. That is, sensory signal in a neural channel—as something which represents—is not just the same as sheer electrical current in that channel. Only a transmission originated by a device selected for sending electrical impulses, e.g. an eardrum or a retina, can count as a sensory signal. And the only way such a device can have gotten selected is if on the historically decisive occasions the impulses it originated attuned other devices in the host organism, ultimately the host's behavioral routines, to some way the environment was (see my [11]). For there is no evolutionary advantage in representing-for-the-sake-of-representing. Representations help just to the extent that they are used, read. The upshot: there is at a time a sensory signal of such-and-such strength in a certain neural channel only if just such a signal is responded to, read. Now suppose that at $t$, in channel $c$, there is a signal of voltage $n$. Then it cannot be that at $t c$ sends a signal of voltage $0.75 n$; these two temporally-indexed features of that channel are contraries. Is that because for a channel to send a signal of $n$ volts, the channel must comprise a part which sends a signal of $0.75 n$ volts, together with another part? But when a channel sends a signal of $n$ volts, no part of it sends a signal of $0.75 n$ volts. There is no signal of $0.75 \mathrm{n}$ volts. For no signal of $0.75 n$ volts is read, responded to. There is no second reading device designed for reading just a part of the channel's signal. There may be electrical current of $0.75 \mathrm{n}$ volts, sent by a part of the neural channel; but electrical current is not eo ipso sensory signal.

If there really are properties such as those considered here-properties 'emergent' in the innocuous sense- then the incompatibility between properties in any one such contrary range cannot be reduced to the Butler impossibility. Each property in such a range excludes all the others, and also differs differently from all the others, and neither the exclusion nor the differing differently can be reduced to the fact that each such property 'is what it is' (read as: has such-and-such internal structure) or 'is not 
another thing'. The very existence of 'emergent' properties involves the obtaining of substantive in re impossibilities.

But are there really any emergent properties? Horsepower is clearly a power, and the other sample properties might plausibly be said to be or involve dispositions. Yet dispositions (and powers) notoriously face ontological challenge. It often is argued that dispositions are not real properties in their own separate right, and that dispositional predicates pick out the non-dispositional, typically categorical, properties which (as one says) 'realize' the dispositions ([2], pp. 72-73; [5], pp. 11-16; [18], pp. 103-107). For after all, it is argued, the latter properties do all the causal work that might ever be attributed to dispositions. If all emergent properties are dispositional, then—as my examples may seem to suggest—it may after all be true that all incompatibilities between real properties do reduce to the Butler impossibility.

In response to this challenge I will argue that in some cases the presence of a dispositional property can causally explain an effect which the presence of the non-dispositional property, which realizes (or replaces) that property, does not explain. So there is nothing ontologically suspect about dispositions per se-if particular dispositional properties demand reduction or replacement, it is not on account of their being dispositions.

But it is important to consider the right sort of case. To say that an object is such that, when $i$ (for 'input') happens to it, it responds by doing $o$ (for 'output')—-that is, to say that the object has such-andsuch a dispositional property-is not to identify what causally explains its doing $o$ in those circumstances. A disposition does not cause its own display. If a disposition has a causal basis, if it is realized by some non-dispositional property or properties — as all dispositional properties arguably are - then what causes its display is precisely that non-dispositional property (or properties) ([21], p. 255). So the only way a disposition might plausibly be said to cause something not caused by the non-dispositional property that 
realizes it is if it causes something beyond the output which is definitory of its occurrence. For this reason, having a valence of +3 does not yield a case which illustrates my thesis. Whatever appears to be explained by an atom's having valence +3 can equally well—actually, better-be attributed to that atom's having the structural features that underlie its valence. (In fact, as I have remarked, these features appear to be as emergent as the valence which they underlie, and if so the causal impotence of valence affords scant comfort for the philosopher who wants to reduce all incompatibilities between real properties to the Butler impossibility; but let this pass.) For the same reason, having horsepower of 409 may not yield a case which illustrates my thesis. For it may be that all that the presence of that horsepower appears to cause is acceleration at such-and-such a rate of such-and-such a mass when the throttle is open. But that is surely caused also-actually, instead—by the configuration and construction of the engine which underlie its having that horsepower.

If dispositions are sometimes causally efficacious, it is most likely that this happens in the realm of biology. For that tokens of a particular biological device are such as to do a certain job in certain circumstances can catch the eye of natural selection, and bring it about, not that their job gets done by them, but that those devices get more and more widely replicated. Or so we do seem to say. What for example has causally explained the spread throughout a common species of frog (Rana pipiens) of neural circuitry which, whenever a fly appears before the frog's eyes, sends a signal that activates neural routines for gulping? The circuitry is indiscriminate. It sends the same signal whenever any small dark object crosses the frog's visual field, even a BB. But what caused the genes for such circuitry to win out over their alleles seems clear enough: on enough occasions, the circuitry launched gulps that eventuated in nutritious items' (specifically, flies') being present in the host's digestive system ([11]). Now note that this explanation appeals to two dispositions, in serial fashion. It says the gulp-launching disposition of the circuitry caught the eye of natural selection just because nutritiousness in some items gulped caught the eye of the gulp-launching disposition. That the items ingested on those occasions were nutritious helps causally explain why the dispositions (tokens) caused reproductive advantage for the genes which had formed them. 
It is on the nutritiousness of those historically ingested flies that my argument first focuses. Is there a non-dispositional property of the flies—e.g. a biochemical one-which might be said really to have done all the causal work that their nutritiousness appears to have done? Suppose for the sake of argument that there is. Suppose it is a perfectly ordinary property - that it has its own proper contraries. Suppose, that is, that we can specify the biochemical composition of those flies in a way that permits us to say, in specific biochemical terms, what it would have been like for a slightly different composition to have been present in the items which the historical frogs ingested, or a fairly different composition, or a markedly different composition. Or more realistically, suppose we can specify the composition as a twofold combination of biochemical properties—-such-and-such a molecular structure and such-and-such a molecular composition—so that 'slightly different versus markedly different' is well defined along each of two dimensions. Then each of the two properties will have contraries, and will be eligible to claim a causal role in the selection of the circuitry which launched gulps of the historically unlucky flies. The nutritiousness of those unlucky flies, for its part, will have its own proper contraries. However we choose to measure the nutritional value of the actual unlucky flies, we will know that their actual nutritiousness is flanked by nutritiousness of greater and lesser degree.

The question then is whether any causal role that can be attributed to the nutritiousness of those flies could be awarded to one or another (or a combination) of their biochemical properties. Now in actual science, what typically counts as evidence that $a$ 's having $F$ is not just correlated with $b$ 's having $G$, but causally responsible for it, is something called 'invariance' ([29], pp. 201-10). ${ }^{6}$ That is, variations

${ }^{5}$ It is not easy to identify arguments to the effect that all properties have contraries; endorsement or rejection of that position tends to be a point of departure for discussions of the nature of properties. Probably the best argument I know of is indirect: assuming so enables solution of certain epistemological puzzles, in particular Goodman's 'new riddle of induction' (see my [13] and [15]). There is a direct argument as well, but not to contemporary tastes—namely in [17], pp. 158-85.

${ }^{6}$ I offer a philosophical defense of counting such invariance as evidence of causality in [15]. 
from $F$ in the property that characterizes $a$-replacement of $F$ by one or another of $F$ 's contraries, in our terms - couple reliably with commensurate variations from $G$ in the property which $b$ displays. In other words there is a constant tracking or 'invariance' tying the determinable to which $G$ and its contraries belong, e.g. degree to which the gulping routines bestow reproductive advantage, and the determinable to which $\mathrm{F}$ and its contraries belong, e.g. degree of nutritiousness in the items ingested.

Now this much seems uncontroversial: distance measured in degree of nutritiousness, from the actual nutritiousness of the items ingested by the early ancestors of today's frogs, would have gone with distance from the degree of reproductive advantage bestowed, by the gulping dispositions, on those ancestors. Indeed the point is not just a counterfactual one. Undoubtedly some actual forerunners of today's frogs did gulp down items of less nutritional value, on average, than today's frogs do, and did suffer reproductive disadvantage as a result. But what about distance measured in biochemical terms from the biochemical composition of the items ingested by the ancestors of today's frogs? Do we have reason to think that if items just a bit different biochemically had been ingested by ancient frogs, just slightly less reproductive advantage would have flowed from the gulping dispositions? Quite possibly not. Replace a favored food with something which differs just slightly along the axis of molecular structure, and you may replace it with something nutritionally worthless. If, for example, the amino acids in the historically unlucky flies—-which are all 'left-handed' molecules—-had been replaced by 'righthanded' mirror images, the historical frogs which gulped them would have derived no benefit at all. Then too, replace a favored food with something which differs markedly along the axis of molecular

${ }^{7}$ One familiar example of how different the results can be of ingesting left- and right-handed versions of the same molecule is provided by the ghastly case of thalidomide, which in its R-form harmlessly reduces nausea during pregnancy, and in its L- form produces birth defects. (The drug was originally marketed in a form which combined both versions.) An example from what humans ingest, of a dramatic difference yielded by a slight difference in molecular composition, is the case of ethanol (C2 $\mathrm{H} 5 \mathrm{OH})$ - harmless in moderation—and methanol $(\mathrm{C} \mathrm{H} 3 \mathrm{OH})$. 
composition, and you may have provided something else of high nutritional value. (Nutritiousness is 'multiply realizable' in biochemical terms.) So biochemical distance from the historical targets of ancestor gulping dispositions may not be matched by commensurate distance from the evolutionary advantage bestowed by those dispositions. But nutritional distance from those same targets assuredly is. The presumption must be that the nutritiousness of the flies causally explains something which the nondispositional character of flies, underlying that nutritiousness, does not.

The same argument applies to dispositions of biological devices themselves, and not just dispositions of their normal targets or tools. Consider for example analogous bodily structures—samelooking structures brought about by 'parallel adaptation' in two or more independent lineages (e.g. wings in birds and insects). Tokens of such a structure typically differ markedly in many non-dispositional respects: having been shaped or exapted from different pre-existing bodily structures, they typically differ in physical composition from lineage to lineage, and are produced by radically different genetic blueprints. The ancestor tokens from which the current ones were copied differed in those same nondispositional respects. But the ancestor tokens were alike in being such as in favorable circumstances to do a common useful job. Their having this disposition brought it about, not that that job got performed, but that those ancestors got replicated, that the lineage-spanning type of structure proliferated. So dispositional properties of such lineage-spanning devices do causal work which cannot be attributed to genuine-i.e., non-disjunctive-properties of those devices. Now true enough, question sometimes is raised whether kinds unified by mere parallel adaptation should be regarded as genuine or natural kinds at all ([19]) — and if not, dispositions characteristic of such 'kinds' can hardly be credited with causal efficacy. But while lineage-spanning kinds support fewer inductions than kinds unified by physical composition and microstructure, they do support some, and can be regarded as natural kinds ([8], pp. 124$129 ;[14]$, p. 196). 
It is very likely that there are in the world emergent properties. If there are, then the 'partial identity' analysis is not in general adequate for explaining the incompatibility of contraries. There is more to this incompatibility than the Butler impossibility. The very existence of properties in the world involves the obtaining in re of substantive modal facts. A realist about modality needs only an ontology of objects and properties $\stackrel{8}{8}$

\section{University of Connecticut}

Received: April 1998

Revised: October 1998

${ }^{8}$ I am grateful to John Troyer, Ruth Millikan, David Armstrong, and Austen Clark for conversations about the topics of this paper, and to Gary Epling, Jane Knox, and James Bobbitt of my university's Chemistry Department for help with the scientific examples. 


\section{REFERENCES}

1. Kathleen Akins, 'Of Sensory Systems and the "Aboutness" of Mental States', Journal of Philosophy, 93 (1996), pp. 337-72.

2. David Armstrong, A World of States of Affairs (Cambridge: Cambridge Univ. Press, 1997).

3. ___ 'Are Quantities Relations? A Reply to Bigelow and Pargetter', Philosophical Studies, 54 (1988), pp. 311-15.

4. ___ Universals and Scientific Realism, Vol. II: a Theory of Universals, (Cambridge: Cambridge Univ. Press, 1978).

5. __ Belief, Truth, and Knowledge (Cambridge: Cambridge Univ. Press, 1973).

6. John Bigelow, Brian Ellis, and Caroline Lierse, 'The World as One of a Kind: Natural Necessity and the Laws of Nature', British Journal for the Philosophy of Science, 43 (1992), pp. 371-88.

7. John Bigelow and Robert Pargetter, Science and Necessity (Cambridge: Cambridge Univ. Press, 1990).

8. Ned Block, 'Anti-Reductionism Slaps Back', in [26].

9. Austen Clark, A Theory of Sentience (Oxford: Oxford Univ. Press, forthcoming).

10. L. Jonathan Cohen, and Mary Hesse, eds., Applications of Inductive Logic (Oxford: Oxford Univ. Press, 1980).

11. Crawford Elder, 'What vs. How in Naturally Selected Representations', Mind, 107 (1998), pp. 34963.

12. ___ 'What Sensory Signals are About', Analysis, 58 (1998), July.

13. ___ , 'Contrariety and "Carving Up Reality”, American Philosophical Quarterly, 33 (1996), pp. 277-89.

14. ___ 'On the Reality of Medium-Sized Objects', Philosophical Studies, 83 (1996), pp. 191-211.

15. ___ 'An Epistemological Defence of Realism about Necessity', Philosophical Quarterly, 42 (1992), pp. 317-36. 
16. , 'Realism, Naturalism, and Culturally Generated Kinds', Philosophical Quarterly, 39 (1989), pp. 425-44.

17. G.W.F. Hegel, The Logic of Hegel, trans. William Wallace (Oxford: Oxford Univ. Press, 1873).

18. J.L. Mackie, 'Dispositions, Grounds, and Causes', in [26], pp. 99-107.

19. Ruth Millikan, 'Historical Kinds and the Special Sciences', forthcoming.

20. Language, Thought, and Other Biological Categories (Cambridge, Mass.: M.I.T. Press, 1984).

21. Elizabeth Prior, Robert Pargetter, and Frank Jackson, 'Three Theses about Dispositions', American Philosophical Quarterly, 19 (1982), pp. 251-57.

22. Denis Robinson, 'Epiphenomenalism, Laws \& Properties', Philosophical Studies, 69 (1993), pp. 134.

23. Sydney Shoemaker, 'Properties and Inductive Projectibility', in [10], pp. 291-312.

24. Sydney Shoemaker, Identity, Cause, and Mind (Cambridge: Cambridge Univ. Press, 1984).

25. Chris Swoyer, 'The Nature of Natural Laws', Australasian Journal of Philosophy, 60 (1982), pp. 203-23.

26. James Tomberlin, ed., Philosophical Perspectives, 11: Mind, Causation, and World (Atascadero: Ridgeview, 1997).

27. Raimo Tuomela, ed., Dispositions (Dordrecht: D. Reidel, 1978).

28. Ludwig Wittgenstein, 'Some Remarks on Logical Form', Proceedings of the Aristotelian Society, supp. vol. 9 (1929), pp. 162-171.

29. James Woodward, 'Realism About Laws', Erkenntnis, 36 (1992), pp. 181-218. 